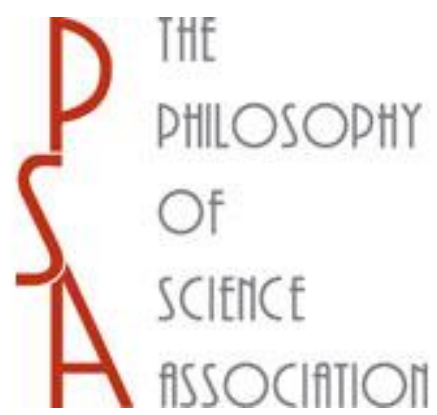

Holism, Individualism, and the Units of Selection

Author(s): Elliott Sober

Source: PSA: Proceedings of the Biennial Meeting of the Philosophy of Science Association, Vol. 1980, Volume Two: Symposia and Invited Papers (1980), pp. 93-121

Published by: The University of Chicago Press on behalf of the Philosophy of Science Association

Stable URL: http://www.jstor.org/stable/192588

Accessed: 30-06-2018 17:53 UTC

\title{
REFERENCES
}

Linked references are available on JSTOR for this article:

http://www.jstor.org/stable/192588?seq=1\&cid=pdf-reference\#references_tab_contents You may need to log in to JSTOR to access the linked references.

JSTOR is a not-for-profit service that helps scholars, researchers, and students discover, use, and build upon a wide range of content in a trusted digital archive. We use information technology and tools to increase productivity and facilitate new forms of scholarship. For more information about JSTOR, please contact support@jstor.org.

Your use of the JSTOR archive indicates your acceptance of the Terms \& Conditions of Use, available at http://about.jstor.org/terms

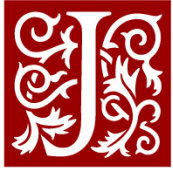

Philosophy of Science Association, The University of Chicago Press are collaborating with JSTOR to digitize, preserve and extend access to PSA: Proceedings of the Biennial Meeting of the Philosophy of Science Association 


\section{Holism, Individualism, and the Units of Selection ${ }^{1}$}

\section{Elliott Sober}

University of Wisconsin, Madison

\section{Holism and Individualism}

The units of selection problem, as it is discussed within evolutionary theory, recapitulates some important elements in the dispute between methodological holism and methodological individualism. Holism and individualism have for a long time occupied favored positions in the stable of old warhorses owned and operated by philosophers of social science. These particular old warhorses are thought by many to be in retirement, although there is less than universal agreement about whether holism or individualism won the battle. Part of the point I will make about group versus individual selection is that biologists would do well not to emulate certain aspects of the holism/individualism controversy. That they have done so already is a point that I will attempt to establish. And, conversely, the substantive empirical issues involved in the units of selection controversy suggest that the holism/individualism debate within the social sciences can be reformulated in a way that makes it nontrivial and also not decidable a priori. This offers some hope that the holism/individualism dispute need not remain a dismal philosophical problem of the "dismal sciences".

Holists and individualists disagree over whether social wholes are more than the sum of their parts (see Brodbeck [1968] for representative essays). Holists say they are and individualists say they are not. There is the appearance of a disagreement here. But the appearance starts to appear illusory when one asks what each side means by "sum"; exactly what is meant when it is asserted, or denied, that the whole is more than the sum of its parts?

Holists are concerned to avoid the sin of atomism. They do not think that social entities can be understood by taking individuals in isolation from each other. To understand social wholes, they insist, one must consider individuals in their relationship to each other and to the environment. When holists assert that the whole is more than

PSA 1980, Volume 2, pp. 93-121

Copyright (C) 1981 by the Philosophy of Science Association 
the sum of its parts, they mean that properties of the whole are not determined by the unary, nonrelational, properties of the parts. To me, the point that holists are making is a truism. That relational properties must be taken into account seems obvious. It looks like this point is true, not just of social objects, but of any object which has parts. One might even suspect that the principle is a priori, or as a priori as anything can be.

Do individualists seriously propose to ignore relations? Are individualists really such benighted atomists? Not at all, say the individualists, who insist they not be confused with the straw man just discussed. Individualists concede that social facts cannot be understood by taking individuals in isolation from each other. But the crucial point is that the character of the whole is fixed by the properties and relations of its parts. The whole is nothing above and beyond those interactions among individuals. Individualists will deny being atomists. What accusation might they hurl back at the holists? Holists, they say, hypostatize (reify) social wholes. Holists, according to this indictment, think that properties of social wholes are not determined by the interactions that individuals have with each other and with the environment. Where, then, does this independent existence of social wholes come from? What is the secret added ingredient one must add to individuals, the environment, and their interactions to get social facts? Holism, thus construed, looks like old-fashioned vitalism; it isn't that some mysterious fluid must be added to matter to get life. Rather, holism is portrayed as holding that you must add some sort of occult social fluid to individuals and their interactions to get social groups.

This time it is the individualists who seem to be right. Reification is precisely what holists are up to, if they believe that the whole is not determined by its parts and their interactions with each other and the environment. Individualism does seem to be correct in claiming that properties of wholes are determined by properties of parts, in this sense.

So what has happened to this dismal dispute? One could embrace atomism on the one hand or hypostatis on the other, and doubtless there have been social scientists who have done so, in practice if not in theory. But if one rejects both of these alternatives, there seems to be no issue left. Yes, in one sense, the whole is more than the sum of its parts, but, in another sense, no, it is not. What else is there to talk about?

I will mention three problems which retain their interest in the face of these truisms. The first is epistemological. One can agree with the truisms and still wonder what the most fruitful research strategy might be in understanding a particular social phenomenon. Even if the truisms are true, it is still an open question what facts would be most interesting to look at in trying to understand the stock market crash of 1929; this social fact may be managable from a macroscopic perspective and completely intractable from a more individualist 
point of view (see Sober 1980 for the relevance of this distinction to the biological species concept). A second sort of question, also untouched by the truisms, concerns the amount of complexity and interaction among parts that needs to be taken into account to explain social facts. Historically the difference between self-styled holists and self-styled individualists has often concerned this question; the issue has not been whether the whole is reducible to the interaction of its parts, but in what ways the determination works (Wimsatt 1980 argues the centrality of this issue to the units of selection problem). A third question which is not addressed by the truisms can be grasped by distinguishing type from token. ${ }^{2}$ What I have been discussing so far is the way in which single social events, like the stock market crash of 1929, are the upshot of interactions among individuals. That is, I have been talking about token social facts. A rather different question concerns the nature of various kinds of social facts. Here, one asks not for an explanation of a particular historical event, but of the nature of a social property. What is capitalism? What is a stock market crash? These questions about types may or may not be answerable in terms of individuals and their interactions. I raise these three questions only to set them to one side.

The truisms, then, do not concern how we might best attempt to understand a particular social fact, but concern that fact's causal connections with the world of individuals. The truisms do not concern the character of social properties, like the nature of crises or production in general, but the causality of single token events, like the stock market crash of 1929. Each whole is determined by interactions among its parts. This truism would be vouchsafed if causality were transitive. I'll assume that it is. 3 The stock market crash was caused, we might suppose, by interactions among various market conditions, which were themselves social facts. These social facts, in turn, obtained because various individuals did what they did in various physical environments. So the interactions among people caused the crash. If this is right, then it is an ill-conceived question which asks: "What caused the crash -- was it the market conditions or was it the way people acted?" One should respond to this question by asking: "What are you saying or for?" There is no asymmetry here. Causality, in virtue of its transitivity, gives aid and comfort neither to the holist nor to the individualist. The causal chain just keeps rolling along.

In what follows, it will be argued that the dispute about the units of selection resembles the holism/individualism dispute, but with the concept of natural selection replacing the more general idea of causality. This replacement makes all the difference in the world, however. Holists and individualists are, or should be, driven to disgruntled agreement by considerations that are not specifically sociological and appear to be almost a priori (like the fact that causality is transitive). In contrast, group selection and individual selection hypotheses admit of no such easy resolution; this dispute, properly construed, turns out to be an interesting empirical and specifically biological one. What is more, the lack of asymmetries just noted, when a question of social versus individual causation is broached, are 
replaced by asymmetries aplenty. It is a very real question to ask "Was a particular social characteristic caused by group or by individual selection?"

\section{Historical and Conceptual Background}

I just argued that holists and individualists interpret the cliche "the whole is more than the sum of its parts" in two different ways. The interpretations given a priori bias the case: holists interpret the slogan in such a way that it cannot fail to be true, whereas individualists tend to understand it in such a way that it cannot fail to be false. This same situation obtains, in much less virulent form, in the unit of selection controversy. I will address here the dispute between group and organismic selectionists, leaving to one side the issues raised concerning genic and molecular selection at one end of the spectrum, and interspecies and community selection at the other (although a brief comment will be made about this in section 6). Authors who believe that group selection has played a relatively minor role in evolution tend to use a definition of group selection that is extremely restrictive; authors who attribute greater potential importance to this selective force often use a more liberal, permissive, conception of group selection. What is more, each position has fairly cogent criticisms of some of the ideas on the other side.

The philosophical focus of this paper is on determining what group selection is; the hope is that we can then use this clarification to pinpoint what distinguishes group and individual processes generally. Yet, it is well to remember that biologists do not have this as their motivation for thinking about group selection, nor did they become interested in group selection as an idle conceptual exercise. The historical context for the recent incarnation of the group selection controversy is that group selection was hypothesized as an explanation of phenomena that allegedly could not be explained in any other way. In 1962, V. C. Wynne-Edwards published his book Animal Dispersion in Relation to Social Behavior. There, he argued that certain adaptations found in nature would be counterpredicted if individual selection were the only selective force at work. Wynne-Edwards talked about the ways in which prey populations react to the approach of predators. He discussed territoriality. He devoted a great deal of attention to the idea that organisms limit their own reproduction when the population approaches the environment's carrying capacity. Each of these categories involves traits which he thought were altruistic: organisms possessing such traits diminished their own reproductive chances while enhancing the fitness of the group. Altruism is always at a disadvantage when compared with selfishness, as far as individual selection is concerned. But groups of altruists may do better than groups of selfish individuals, and this, Wynne-Edwards argued, explains why altruism is such a common and stable phenomenon in nature.

Four years later, George C. Williams published his Adaptation and Natural Selection. Williams subjected group selection to two lines of attack. First, he looked at the alleged examples of altruism that 
Wynne-Edwards had discussed and argued that they could be analyzed differently. Sentinel crows issue alarm cries when a predator approaches. Wynne-Edwards saw this as an example of altruism; the sentinel places itself in peril for the good of the group. But a number of alternative, individualist, construals can be offered: perhaps the sentinel's warnings benefit its own offspring more than they benefit unrelated individuals. If so, the issuing of warning cries is, like parental care, perfectly consistent with the selfish calculus of individual selection. Or perhaps, though the warning cries are heard by related and unrelated individuals alike, the accoustical properties of the cry do not expose the sentinel to increased risk; maybe predators can't localize them. Or, perhaps the cries have the effect of causing a flurry of activity in the flock, and thereby conceal the sentinel from the approaching predator.

The occurrences of "perhaps" above deserve notice. The empirical details of sentinel crows, and of the other phenomena that Williams and Wynne-Edwards discuss, are incompletely understood. What Williams was doing was not providing known facts which, in every instance, refuted Wynne-Edwards' suggestions. Rather, he was telling an alternative story which was capable of explaining the observations from the point of view of individual selection alone. So given our paucity of details about sentinel crows and the other examples that Wynne-Edwards and Williams discuss, we might say that there are two possible explanations, at least, of the observations. One of them is provided by Wynne-Edwards' group selection hypothesis, the other by Williams' individual selection hypothesis. Do we have here a stand-off? According to Williams, we do not, since it is more parsimonious, he says, to invoke individual selection alone to account for these controversial cases.

Since I have discussed the principle of parsimony and its application to the group selection controversy elsewhere (1981b), I will not go into a great deal of detail in discussing the merits of such arguments in general or of Williams' argument in particular. However, a few comments are in order. One, not uncommon, reaction to Williams' parsimony argument is to dismiss it in such a way as to imply that considerations of parsimony never count for anything. As one biologist said to me: "The fact that Williams doesn't need group selection has nothing to do with whether group selection exists." If the thought behind this remark were true, then Ockham's razor would be a purely aesthetic consideration, never offering us a reason for thinking that a given hypothesis is true.

I do not take this wholly negative view of Williams' parsimony argument, although there can be no doubt that parsimony alone does not place individual selection on a thoroughly satisfying theoretical basis. One of the limitations of parsimony arguments in general is that they do not offer us an explanation of why the parsimonious hypothesis is true. Maybe we ought to believe that individual selection better accounts for the phenomena that Wynne-Edwards discussed. But this does not explain why group selection has played so minor a role in the history of evolution. Sewall Wright (1978) discusses this point. He suggested 
to place the subject on a more secure foundation, we must create quantitative models of group selection, determine under what parameter values group selection would be efficacious, and then go to nature to see when, if ever, those parameter values are satisfied. Then we would have a more substantial reason for being parsimonious; we also would have an explanation of why group selection never, or rarely, occurs.

I believe that this limitation of parsimony arguments has the curious result of revealing why they can have a rational basis. Parsimony arguments are inductive arguments. Induction from a sample to a containing population does not provide one with an explanation of why the containing population is as it is. Williams wanted to explain the controversial phenomena over which he and Wynne-Edwards differed in a way in which everyone agreed the noncontroversial phenomena were to be explained. What was uncontroversial (at least to the participants in this dispute) was that individual selection was the mechanism behind numerous adaptations. Williams' parsimony argument was simply the assertion that we should use old mechanisms to explain new phenomena. Since the sampled, already understood, adaptations were due to individual selection, we infer that the new cases at issue are caused by the same thing.

Inductive arguments, I assume, can, if they are any good, provide us with reasons for believing their conclusions. So the issue of the quality of Williams' parsimony argument reduces to the issue of whether his inductive argument is strong. Are the known cases representative of the kinds of adaptations found in nature, or do they involve a biased sample? This and other questions are suggested by Wright's (1978) evaluation of the parsimony argument. What they show is that parsimony arguments can provide us with reasons, if they meet the standards of good induction. But even when they do this, they are always incomplete, from the point of view of a science which has explanation as a goal.

As I mentioned above, Williams offered a second line of argument which is supposed to count against the importance of group selection. This involved quantitative considerations based on Fisher's (1930) socalled Fundamental Theorem of Natural Selection. The upshot of these considerations (also discussed in Lewontin 1970) is that group selection will probably play a restricted, and relatively minor, role in the history of evolution, when compared with individual selection. These quantitative arguments do not show that group selection never exists, or that it couldn't exist. As we'll see, Williams believed that at least one real case of group selection has probably been found in nature, in any case. Although these quantitative considerations are separable from our goal of describing what group selection is, the definition we will arrive at has some ramifications for the quantitative question.

Wynne-Edwards' work and Williams' attack were followed by a series of theoretical papers (reviewed in wade 1978) in which mathematical models were proposed and examined. The main result of these analyses has appeared to confirm Williams' orientation, in that the parameter values needed for group selection to have significant impact were generally 
found to be quite restrictive. This conclusion, however, has not gone unchallenged, in that it is arguable that the models contain several unrealistic assumptions that a priori bias the case against group selection (Wade 1978). In my opinion, the quantitative question remains open.

As I have indicated, our interest here is not in the issue of how much of a difference group selection has made, but rather in the question of what group selection is. The various arguments and approaches that have fueled the biological controversy suggest that some rather different conceptions of group selection have been at work. Before we can identify these points of divergence, however, we ought to be clear on the common conceptual structure which is not in dispute.

For natural selection to act on a set of objects, there must be variation -- the objects must be different. Moreover, the differences between the objects must include differences in their probabilities of reproductive success -- there must be variation in fitness. And lastly, it usually is assumed that the fitness of parents must be correlated with the fitness of offspring -- there must be heritable variation in fitness (adapted from Lewontin 1970). This last requirement seems to me to be inessential for the existence of natural selection, although it is essential if cumulative genetic evolution by means of natural selection is to take place.

A word of clarification is in order concerning how the concept of fitness will be understood here. In any model of evolutionary processes which accords a role to random drift, fitness cannot be defined as actual reproductive success (e.g., number of viable offspring). Since any realistic model must give drift its due, fitness is not identical with actual reproductive success. The so-called tautology of the survival of the fittest is no tautology at all; the fitter do not always turn out to be more successful. The natural reaction to this fact is to think of fitness as an expectation, in the mathematical sense, of reproductive success (see, e.g., Crow and Kimura 1970, p. 178, Mills and Beatty 1979, and Sober 1981a for discussion). The fitness of an object is its propensity, or disposition, to be reproductively successful. Fitness differences, thus construed, may be the causes of reproductive differences.

The conditions set out above for natural selection to act on a set of objects -- namely that the objects should vary in fitness -- require supplementation to avoid the following problem. Consider a set of organisms which are causally isolated from each other; they may be at opposite ends of the universe and experience entirely different kinds of environmental stress. Suppose they are different in their fitness values. Still, it would be odd to conclude that there is a selection process in which they are all involved. One solution to this problem is to require that the objects be in competition with each other. But, as Lewontin (1978) has argued, this is inessential; two bacterial strains may be subject to natural selection even when neither impinges on the other's access to resources. The strains, growing in an excess 
of nutrient broth, may have unlimited energy available, but selection may favor the strain with the faster division time. As Darwin remarked, "A plant at the edge of the desert is said to struggle for life against the drought." By implication, selection can favor the plant better suited to the desert conditions, even when the better and worse plants do not interfere with each other. To be sure, competition is a familiar way of thinking about natural selection; yet, curiously, the familiar cases of natural selection that serve as textbook examples do not involve competition. The evolution of industrial melanism and of immunity to DDT do not involve there being a common resource in short supply. Competition is a special case, not a defining characteristic, of natural selection.

A more general conception of what subsumes a set of objects under a single selection process is that there must be some common causal influence acting on the objects which affects their reproductive chances. This common influence I will call a force. Much latitude exists for determining whether two objects are subject to the same force. It may be appropriate to think of the organisms in geographically isolated local populations of the same species as all involved in a single selection process. If each experiences predation as its major environmental problem, this may suffice to say that they are exposed to the same force. If, however, some experience predation, others experience temperature fluctuation, and still others experience the disappearance of prey as the major environmental stress, it will be wrong to lump these organisms together and talk about a single selection process subsuming them all. The sameness of the forces impinging on different organisms will be determined not just by the physical characteristics of the environment, but also by the biology of the organisms involved. If a field is sprayed with one insecticide, and a second field is sprayed with a second insecticide, it may be perfectly correct to construe the two affected insect populations as part of the same selection process. This will be true if the physical differences in the insecticides make no difference in the way those chemicals impinge on the organisms. The idea of "sameness of force" needs to be read biologically. 4

So far, I have talked about a set of objects satisfying certain conditions. What are these objects? How should the abstract structure of these conditions be interpreted? The classical, Darwinian, interpretation is that the objects are organisms that exist within the same population. Organismic, or individual, selection is generally understood as this sort of within group selection. Group selection, on the other hand, involves interpreting the structure so that the objects involved are groups. Groups differ in their capacity to contribute to the next generation. Group reproduction is here understood to require the founding of numerically distinct colonies; mere growth in size of the group isn't enough. So, to get started in considering what group selection is, imagine a set of groups which differ from each other in their expectations of reproductive success. Group selection will thereby involve selection between groups, whereas individual selection involves selection within groups. 


\section{The Artefact Argument}

Can one define group selection in the way just suggested, as existing whenever there is heritable variation in the fitnesses of groups? I would say not, although some biologists have used this sort of permissive characterization. The defect of the definition is that differences in reproductive capacity that obtain between groups may merely be artefacts of the differences in firtness that obtain between organisms. Williams (1966) again and again deploys this idea in criticizing group selection hypotheses. The fact that natural selection has the effect that some groups are more reproductively successful than others is not enough to show that one has group selection. Selection at lower levels of organization can have this sort of "macroscopic" upshot.

Williams' artefact argument, as I will call it, asserts that the mere existence of differences in group productivity, or in group fitness, is not enough to demonstrate that there is group selection. The crucial question is where those differences came from: are they an artefact of selection processes occurring at other levels, or are they due to selection occurring at the level of groups? The distinction being made here must be spelled out in an adequate characterization of what group selection is. 5

This line of thinking has its counterpart in the methodological holist versus methodological individualist controversy. Suppose holists argued that their position is confirmed by the fact that some social event, like the stock market crash of 1929, was caused by the occurrence of certain market conditions, which are themselves social events and states of affairs. Individualists might grant the causal claim, but argue that this by no means argues in favor of holism. After all, it still is possible, and indeed is to be expected, that individual interactions brought about those very market conditions. That social facts are causally efficacious does not show that their causal efficacy is irreducible. Individualists will often demand to be shown how social facts can exist without an individualist foundation. Williams, in his criticisms of group selection hypotheses, demands to be shown cases in which the causation of group properties is not reducible to individual selection.

Let's illustrate how the artefact argument works with a simple example. Imagine a system of populations, each of which is internally homogeneous with respect to height. All the individuals in population $\# 1$ are 1 foot tall, all those in population \#2 are 2 feet tall, and so on, for six such populations. Now imagine that natural selection favors individuals which are taller over ones which are shorter. As a result, population \#6 will be more reproductively successful than population \#5, and so on. Is this a case of group selection? I doubt that many biologists would want to say that it is, and I am certain that Williams' artefact argument entails that it is not. What one has here is a case in which the differential reproductive success of groups is an artefact of differences in individual fitness. Group selection isn't to be equated with there being heritable variation in the fitnesses of groups. 6 
One of the assumptions underlying Williams' artefact argument is that group selection and individual selection are objectively distinct forces of evolution; in the above case, the group selection description is false while the individual selection description is true. Now it is conceivable that one might give up this assumption and view the concepts of group and individual selection as interchangable and equivalent, the way that some positivists have viewed the relationship between Euclidean and non-Euclidean geometry. In this vein, one might hold that whenever there is heritable variation in the fitness of groups, you can say that there is group selection or not, as you please. The choice would be one of convenience, in that both descriptions would be correct. According to this view, group selection and individual selection are not related to each other the way that mutation and migration are related to each other, namely, as two objectively distinct observerindependent forces of evolution.

Actually, this "conventionalist" attitude is not just conceivable, but is suggested by one of the oldest and most influential models of group selection, that of sewall Wright (1931). Wright postulated a system of semi-isolated local populations. In virtue of their small size, random drift has more chance to operate, so that an allele might drift to a sufficiently high frequency for individual selection to take over and then drive the trait to fixation. The population would then send out migrants who would make over other local populations by the same process. Is this group selection? Well, it can be described as a case in which one has drift acting on individuals within a population, individual selection, and migration of individuals. One could, I suppose, define group selection as existing whenever these three individual-level processes occur. But then group selection is not a distinct evolutionary force, and Williams' artefact argument cannot be made.

\section{Context Sensitivity of Fitness}

How are we to strengthen our definition of group selection? What more is there to group selection beyond there being heritable variation in the fitness of groups? One natural suggestion is this: if the change in gene frequency one gets would not have happened if one had a single panmictic population, then one has group selection. That is, returning to our example of the populations distinguished by the heights of their inhabitants, we get this result: if the differential reproductive success that obtains in this situation differs from what would have happened if there had been a single interbreeding population made up of all the individuals, then we've got group selection. The idea here, which has obvious application to Wright's model, is that group selection exists whenever population structure affects reproductive success. A central point of Wright's model was to show how a system of small semi-isolated local populations could undergo evolutionary changes that would be extremely improbable in the kind of large panmictic population that R. A. Fisher (1930) considered.

Is this an adequate definition? Can one say that group selection exists whenever there is heritable variation in the fitness of groups, 
where those fitness values depend on population structure? I'll set to one side the problem of figuring out the character of the counterfactual situation we are supposed to consider; if the groups exist in different environments, what are we to imagine is the environment that the hypothetical single panmictic population occupies? This problem aside, it seems clear that the proposed definition is extremely liberal, in that it makes group selection a commonplace of evolution. It is a virtually universal fact about fitness that an organism's fitness depends on what the other organisms are like in the population. Thus, whenever one has a system of populations, one can expect individual fitnesses to depend in part on the way in which organisms are distributed into populations. In particular, one can expect the fitness values to differ from what they would be if there were a single panmictic population. Does this suffice for group selection? I would say not; group selection must involve more than the fact that fitness values are context sensitive.

Let me report a classic finding concerning this fact of context sensitivity. Levene, Pavlovsky, and Dobzhansky (1954) found that the socalled Arrowhead homozygote on the third chromosome of Drosophila pseudoobscura is fitter than the Chiricahua homozygote, under laboratory conditions in which just these two chromosome types competed. However, when a selection experiment was run in which Arrowhead and Chiricahua competed with the Standard chromosome type, Arrowhead turned out to be less fit than Chiricahua. This showed how relative fitness of a trait can depend, not just on the physical environment of the population, but on what other traits are present in the population itself. Now imagine two population cages, one containing Arrowhead and Standard, the other containing Chiricahua and Standard. Fitness values in the two pairwise competitions would be different from what would obtain if Arrowhead, Chiricahua, and standard were all present in a single population. But that there would be a difference in no way implies that the two pair-wise competitions involve group selection. Group selection must involve more than the idea that the fitness values of organisms is influenced by the kind of groups they are in. 7

A parallel line of argument can be traced in the holism/individualism dispute. Holists sometimes argue for their position by citing cases in which the properties of individuals are influenced by the groups to which they belong. We will see later on that certain situations of this kind can be crucial for confirming holism; however, the mere fact that the properties of individuals are context sensitive does not win the day against individualism. For the individualist will simply point out that the group properties which shape the character of individuals are themselves the product of individuals and their interactions. Again, if one is unwilling to hypostatize groups, the individualist's assertion cannot be faulted. For the point being made is simply that the context sensitivity of individual properties -- the way in which they depend on group context -- is perfectly consistent with individuals being the material basis for all higher-level phenomena. 


\section{Altruism}

So far, I have examined two rather permissive definitions of group selection and argued that they are inadequate. I argued for their inadequacy by taking seriously Williams' artefact argument. Group selection is not to be identified with there being heritable variation in the fitness of groups; nor should it be identified with there being heritable variation in the fitness of groups where organismic fitness is context sensitive. Neither of these conditions is sufficient for group selection.

In each of the two proposals, it was found that alleged cases of group selection could be analyzed in terms of individual-level processes alone. This is a standard ploy that individualists use to combat the arguments of holists. A natural response by holists to this line of argument is to try to find some social fact which would be counterpredicted by the assumptions of individualism. If this could be found, then the individualists' strategy of assimilating the phenomenon into their own framework would be blocked. Although phenomena which individualists can accommodate may not count as "real" group processes, ones that they cannot accommodate will qualify as paradigm cases of what holism is all about.

This thought may lead us back to the issue which fueled the fires of the group selection dispute initially. Altruism is counterpredicted by individual selection. 8 Group selection however, might be supposed to promote the existence of altruism, since groups which contain altruists may fare better than ones which do not. So, if one wants to argue for the efficacy of group selection, what better phenomenon to look for than cases in which altruism has emerged and remained prevalent owing to natural selection? Let us be careful here. There are two roles which the concept of altruism might be taken to play in the idea of group selection. One of them concerns how a biologist might try to discover cases of group selection: look for altruism. The other concerns what group selection is (not how we might find out about it); on this view, group selection must be selection for altruism. The difference here is that between an epistemological and an ontological consideration.

The ontological thesis, that group selection must work in a direction opposite to that of individual selection, has one virtue. It has the clear implication that group selection is objectively distinct from individual-level forces like individual selection. It thereby cannot be criticized by invoking Williams' artefact argument; the typical individualist ploy of reanalyzing alleged group-level phenomena as artefacts of individual-level processes has been forestalled.

So, does group selection have to involve selection for altruism? $\mathrm{Be}-$ fore considering some biological examples which show that it need not, I want to note a rather abstract oddity of this idea. It implies that if there is group selection at work on a system of populations, individual selection must be acting as well. That is, we would have here a force 
of evolution which could not act alone. This, by itself, is not a conclusive reason for rejecting the idea, but it does show the proposed definition to conflict with a rather plausible requirement on evolutionary forces, and, perhaps, on all forces in general. It should be possible to describe the changes a force would bring about, if there were no other forces at work. That is, one might suppose that the use of the ceteris paribus condition is not merely permitted when it comes to describing a force, but that it is necessary for the adequate description of a force that this be possible. This corresponds to the idea that a force should be isolatable in principle. I do not propose to defend this idea of isolatability, but merely note that it is rather standard fare in our conception of force. If group selection requires selection for altruism, this condition cannot be satisfied.

But the decisive reason for rejecting this view of group selection is more down to earth and biological. It is simply that some cases which seem clearly to be ones of group selection involve group and organismic selection acting in the same direction. A proper conception of group selection should be tailored to allow for this fact. Let me describe two examples, one of them being the sole case believed by Williams to be a real instance of group selection. Here I mean the investigation by Lewontin and Dunn (1960) of the segregator distorter $\underline{t}_{-}$ allele in the house mouse Mus musculus.

Let me give an elementary description of how the process of segregation distortion, or meiotic drive, works (see Crow 1979 for details). Diploid organisms are ones whose chromosomes come in pairs. In the formation of sex cells, these pairs of chromosomes separate, so that sperms and eggs contain one chromosome each from each pair -- they are haploid. The normal pattern for this reduction is that $50 \%$ of the sex cells contain one chromosome and 50\% the other, from each homologous pair. But when a segregator distorter allele is present on a chromosome, it "subverts" this equality of representation and secures for itself representation of greater than $50 \%$.

This is what the t-allele does in the house mouse. Consider the males who are heterozygote for the $\underline{t}$-allele. One might expect that $50 \%$ of the sperm pool of this group would be made of gametes containing the $t$-allele. In fact, the representation of the segregator distorter is $85 \%$. So at this level there is strong selective pressure favoring chromosomes which contain the t-allele. Let us call this chromosomal selection. Chromosomes having the trait are at an advantage over ones lacking it.

If we go up a level or two, from chromosomes to organisms, the $t$ allele is not favored by selection. Males who are homozygous for the t-allele are sterile. So there is strong organismic selection working against the t-allele.

Lewontin and Dunn combined this information about the chromosomal and organismic selection acting on the $t$-allele and derived a prediction of what the frequency of the $t$-allele should be in nature. It 
was wrong; the prediction erred by being too high. This suggested to them that some third force was acting against the t-allele. The third force was group selection. The population structure of the house mouse is one of small local demes. Whenever all the males in one of these small groups are homozygous for the $t$-allele, the entire deme goes extinct. Females living in a group all of whose males are homozygous will have no offspring. What is more, their fitnesses (or rather the component of fitness determined by this selection process) will be 0 , owing to the fact that they belong to a group of a certain kind. $\mathrm{Fe}-$ males within such a group may differ in phenotype and genotype as much as you like, but such differences make no difference; their reproductive chances have been destroyed by their belonging to the kind of group they're in. Since the frequency of t-alleles among females in such groups will, on average, be higher than the frequency of t-alleles among females in groups lacking this fatal flaw, the effect of group selection will be to reduce the frequency of the $t$-allele. Notice that in this case, organismic and group selection are in the same direction;

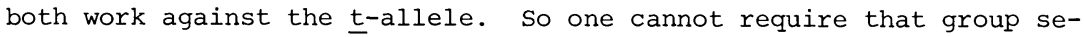
lection and individual selection always be opposing forces.

The other example I want to describe in which individual and group selection work in the same direction is a group selection experiment that Michael Wade (1976) carried out on the flour beetle, Tribolium castaneum. Wade's experiment involved setting up and monitoring four selection processes at once. In each of them, he started with 48 populations, each containing 16 beetles each. At the end of 37 days, he did his selecting. In one of the treatments, he selected for large populations; he located the population containing the largest number of adults and used it to found colonies of 16 individuals each until the population was exhausted. He then went to the next largest group and did the same, until 48 second generation populations were founded. He repeated this process for a number of generations. The average size of populations at the end of the selection process was higher than the average size of the populations in the first generation. Here we have group selection; groups were selected in virtue of their being large.

Another group selection procedure was carried out on a second set of 48 populations. Here, wade selected for small populations. The regimen was as before, and at the end of the procedure the average size was much reduced from what it had been at the start.

A third selection treatment served as a control group. Again, there were 48 canisters. At the end of 37 days, one sample of 16 individuals was drawn from each canister and used to found a next generation population. The only selection process that took place here was within populations. Individuals within a canister competed with each other. But, roughly speaking, each group had the same chance of representation in the next generation as any other; each contributed one and only one colony of 16 individuals. This experimental treatment involved individual selection but no group selection. What happened in the process? Under individual selection alone, the average population size declined, owing to such factors as lengthened developmental time, reduced 
fecundity, and increased cannibalism. 9

Now what happened in the control treatment also happened in the two group selection treatments just described. That is, within each canister in the group selection treatments, individual selection was going on as well. This force, we learn from the control treatment, promoted reduction in population size. In the group selection treatment, in which there was group selection for reduced population size, there were in fact two forces at work. Individual selection promoted reduction in population size, and group selection did the same thing. As one might expect, the magnitude of the reduction that took place in the group selection treatment was greater than that achieved by individual selection alone in the control treatment. Two forces are better than one. Here we have the same lesson as that obtained in the $t$-allele example. Group selection and individual selection can act in the same direction; group selection does not have to be selection for altruism.10

\section{Group Selection Defined}

My purpose in discussing these two examples has not merely been to argue that altruism is inessential. In addition, I wanted to add some data which may serve to constrain an adequate definition of group selection. Let me review the other requirements that a reasonable definition should fulfill. First, the definition should allow one to distinguish changes in group properties due to group selection from changes in group properties that are due to processes occurring at lower levels of organization. That is, an adequate definition should take seriously Williams' artefact argument. Secondly, the definition should not have the consequence that group selection exists whenever fitness values are context sensitive. Group selection does not exist simply in virtue of the fact that the fitness values of organisms depend on the character of the group they are in. And, thirdly, it should turn out that group selection can exist both in the presence or absence of organismic selection and can act in the same or opposite direction from it. This last consideration combines the rejection of altruism as a criterion of group selection with the earlier remarks to the effect that group selection should be an objectively distinct force of evolution.

With this elaborate preamble, the definition can be stated:

Group selection acts on a set of groups if, and only if, there is a force impinging on those groups which makes it the case that for each group, there is some property of the group which determines one component of the fitness of every member of the group.

Let me try to state the intuitive idea in a less cumbersome way. When group selection occurs, all the organisms in the same group are bound together by a common fate. As far as this selective force is concerned, they are equally fit. What determines these identical fitness values (on the component of fitness at issue) is their membership in the same group. Individuals with radically different genotypes and phenotypes may have identical fitness values, owing to their belonging to the 
same group. And individuals with identical genotypes and phenotypes may have very different fitness values, owing to their belonging to different groups. Under group selection, what is causally efficacious in the production of reproductive differences among organisms is membership in groups of different kinds.

Let's apply this idea to the examples discussed so far, starting with the contrived example of populations in which everyone has the same height. Our definition explains why this is not a case of group selection. Although every individual in the same group has the same fitness value, the cause of this sameness is not common membership in a group of a certain kind. A simple explanation is also available of why our two pair-wise competitions between Arrowhead and Standard and between Chiricahua and Standard in adjacent population cages was not a case of group selection. Although the fitness of a fruit fly depended on the kind of group it was in, the members of the same group were not acted on as a unit. The members of the same group did not have the same fitness, on any component of fitness. Rather, group context served to determine the differential fitnesses of organisms with the group, in just the way that the environment can determine fitness differences in cases of ordinary individual selection. Group properties existed, but these failed to ramify back on the fitnesses of organisms in the appropriate way.

Our definition also explains why the two cases of group selection discussed before do really count as group selection. In the $t$-allele example, every mouse has fitness equal to 0 , if it belongs to a group all of whose males are homozygous for the $t$-allele. What is crucial is that this common fitness value is caused by common membership in a group of a certain kind. The same is true of Wade's selection experiment. If there is group selection for groups of a certain size, then every individual in a group has an equal chance of finding its way into the next generation. The individuals within a population are bound together, their common fitness values determined by their common membership in a group of a certain size.

Before moving on to another example of group selection and to some further biological considerations, I want to point out a philosophically interesting feature of the definition proposed. The claim that a set of groups is subject to group selection will differ from the claim that a set of objects is subject to familiar physical forces like gravity or electromagnetism. Group selection may take endlessly many different physical forms; to say that some populations are undergoing a group selection process is not yet to say what physical properties are causally efficacious, but rather is just to say that some physical property or other is responsible for fitness values in a certain way. In contrast, claiming that a particular physical force is acting on a set of objects is a much more specific claim about the physical details; for example, to say that a physical object is in an electromagnetic field is to say that its charge and its distances from other objects play a specific kind of causal role. It is in this sense that claims about evolutionary forces can be more "abstract" than claims about physical forces. This greater degree of abstractness -- this formulation of 
generalizations which are true of objects which differ physically from each other -- is achieved in evolutionary theory by quantifying over properties. Besides the inevitable ontological commitment to numbers which any mathematical theory will involve, evolutionary theory is thereby Platonistic in an additional respect. This is one reason, among others (discussed at greater length in sober 198la) for thinking that a purely extensional ontology will be unsatisfactory for this science.

In order to give the reader some further grasp of the phenomenon that a definition of group selection is supposed to circumscribe, I want to describe another biological example which is often cited (e.g., by Lewontin 1970) as a probable case of group selection. The empirical details to be described have biological plausibility, but they may be revised or replaced by further information. Our interest, though, is not in whether they are true, but in what the assumption of their truth tells us about what group selection is.

Here I have in mind the coevolution in Australia of the disease virus myxoma and the rabbit oryctolagus cuniculus. Myxoma was introduced into Australia to cut down on the rabbit population. Two familiar epidemiological events ensued. On the one hand, rabbits became more immune to the disease; on the other, the virus became less virulent. The explanation of the latter change is that the disease is spread from rabbit to rabbit by a mosquito which only bites live rabbits. Thus, an extremely virulent strain of myxoma, while it may become predominant within a single host, runs a good chance of never spreading through the rabbit population. Less virulent strains, on the other hand, while they succeed in expropriating a smaller number of the host's cells, nevertheless increase their chances of transmission.

Two, opposing, selection forces are at work here. Within each rabbit, strains of greater virulence will tend to consume a greater proportional share of the limiting resource -- namely the host's own cells. So there is individual (within group) selection for increased virulence. But a virus winning this race may thereby lose another -that of spreading its genes to other rabbits. A virus population -the assemblage of different strains within a single rabbit -- founds colonies, and roughly speaking, the lower the average virulence of a population, the better the chances are that a mosquito will transport a colonizing propagule from that population to another host. Assuming that these two selection forces are the main evolutionary forces at work, the fact that the virus declined in virulence shows that in this case the group selection force was stronger than the force of individual selection.

Less virulent strains of myxoma are "a?truists". By being less virulent, they reduce their expectation of reproductive success within the population they are in, but thereby increase the group's chances of survival and reproduction by lowering the average virulence of the population. This example should correct the popular misconception that altruism must always be driven to extinction by a selection process. 
Evolutionary theory entails no such theorem. Rather, what this example shows is that a crucial factor in determining the evolution of a system of this kind, in which group and individual selection oppose each other, is time. If mosquitoes bit rabbits much more rarely, or if myxoma expropriated host cells at a much faster rate, the decline in virulence of myxoma might never have occurred.

There is a theorem which represents this general idea, however. Fisher's fundamental theorem of natural selection (1930) states that the rate of evolution under natural selection is identical to the additive genetic variance in fitness. Since the fundamental theorem has to do with the rate of evolution, evolution will proceed faster, the shorter the generation time of the objects involved. But since groups almost always take longer to found new colonies than the individuals within the groups take to reproduce themselves, one again has the consequence that group selection will produce smaller changes than individual selection (Crow and Kimura 1970, Lewontin 1970). In the myxoma example, group selection was able to exert a powerful influence precisely because of the contingent facts concerning group and individual generation times.

Although I don't want to contest the correctness of applying Fisher's theorem in this case, it is important to identify a presupposition of using it in the general argument that group selection works more slowly than individual selection. It was pointed out earlier that the idea of group reproduction standardly used in discussing group selection is that of groups founding numerically distinct colonies. But there is no need to restrict our attention to this process, to the exclusion of considering the dynamics of population growth. Indeed, the definition of group selection we have arrived at is perfectly consistent with a system of groups undergoing a group selection process in which fitter groups increase in relative numbers. The total number of groups need not change at all. But if this kind of group selection process is considered, the argument based on Fisher's theorem cannot be made. Although individuals usually reproduce faster than their containing groups found colonies, it isn't quite so ubiquitous that individual reproduction takes place in the context of noncolonizing groups which are at their carrying capacity. This point leaves open the possibility, of course, that other broad differences between groups and organisms may be harnessed to Fisher's theorem in support of the claim that group selection is a weaker force of evolution than individual selection (see, for example, the argument of Lewontin 1970 concerning heritability).

Before drawing a few general lessons concerning what the definition of group selection implies about general features of the concepts of fitness and selection, I want to take up an objection to the proposed definition. According to the definition, the members of the same group must have precisely the same fitness values (on the component of fitness at issue) if group selection is at work. But this sounds too strong; for example, group selection might exist simply in virtue of the fact that membership in groups of different kinds had some 
percentage effect on some other fitness parameter. For example, each member of a particular group might have its overall fitness boosted by $5 \%$ by belonging to a group of a certain kind. What is crucial is uniform effect, in some sense; identical fitness values are not required, strictly speaking.

If a property of a group drives predators away, the individuals in the group need not benefit equally. Some might have been better than others in evading predators to begin with, and so the removal of danger may represent an unequal benefit. Still, this may be a genuine case of group selection. If some groups have properties which attract predators while others have properties which repel them, a group selection process may ensue. Though the numerical increments in fitness that members of the same group obtain from the shared group property may be unequal, the fundamental causal structure of a group selection process is still intact. The group's relation to the predator, in this case, is such that the predator reacts to the group as a unit. Although the numbers assigned to individuals may not transparently represent this, the biological relationship of the group to its predator subsumes each individual indifferently. Though fitness values within the group may differ, each individual encounters a predator to the degree that it does because of the property of the group it is in. Whether the biologist characterizes this aspect of the ecology in terms of a separate component of fitness or views it as a partial determinant of some more encompassing component is not what matters.

A number of consequences follow from our discussion concerning the concepts of fitness and selection. As soon as fitness is decoupled from actual reproductive success, it follows inevitably that one cannot read off fitness values from patterns of reproduction. The fact that some groups reproduce more than others does not mean that the more productive groups are fitter. Nor does the fact that some species speciate and persist more than others imply that species selection is occurring, or that some species are fitter than others (see stanley 1975 and Gould 1980 for discussions of species selection). Fitness and selection are both causal concepts; they describe the causes of change and not the fact that there has been differential productivity.

Perhaps a more surprising consequence of our discussion is that fitness and selection are decoupled from each other. In spite of the fact that fitness values and selection coefficients are interdefinable in mathematical models (so that, typically, $\underline{s}=1-\underline{w}$ ), there is an important difference between these concepts. $\bar{A} s$ we saw in our simple example of a series of populations which were each internally homogeneous for height, the fact that groups differ in fitness does not imply that there is group selection. The groups, in this example, differed in fitness in that they had different propensities to be reproductively successful. But the cause of these fitness differences was individual, not group, selection.

Selection is a richer concept than fitness. In fact, the relation of selection to fitness is somewhat like the relation of fitness to 
actual reproductive success. To say that group selection occurs is to say more than simply that groups differ in fitness; it is to say why those fitness differences obtain. Selection is the cause of fitness differences, just as fitness differences may be the cause of differences in actual reproductive success. It follows from this that just as one cannot read off the level of selection from facts about differential productivity, one cannot read off the level of selection from facts about differential fitness. The difference between individual and group selection is not the same as that between within-group and between-group variance in fitness. By the same token, even if some species could be shown to have a greater tendency to speciate, this would not suffice to establish the existence of species selection. The question that remains unanswered is the causal one of why these differences in the expectation of splitting obtain.

This difference between fitness and selection is not surprising, when one considers that fitness is a disposition while natural selection is a force. Although the forces at work determine certain dispositions in the objects present, the dispositions of those objects do not uniquely determine what forces are at work. Thus, to be told that two billiard balls are disposed to accelerate toward each other from their initial positions is not to say what force or forces endowed the objects with that disposition. On the other hand, to specify that the two objects generate an electromagnetic field determines one of their dispositions to move in certain ways.

Our analysis also reveals the inadequacy of two lines of argument that are sometimes offered in defense of lower-level -- either organismic or genic -- selectionism. It is sometimes pointed out that all of the alleged higher-level interactions which may obtain owing to population structure can be given mathematical representation in the fitness values of individual organisms or of individual genes. That is, the effects of processes at higher levels can be viewed as part of the environment of genes, and the whole selection story can be told in terms of the selection coefficients of individual genes. One criticism of this line of thinking is epistemological and, therefore, inconsequential: no one at present knows enough about any gene to define for it a selection coefficient which takes account of all this information. But this line of attack misses its mark since the proposal does not describe what we as theorists can successfully codify, but makes a claim about what is going on in nature.

The fundamental flaw in this kind of argument is that it confuses the task of formulating a predictively successful mathematical apparatus with the task of accurately describing the causal structure of selection processes. It is to be granted that all of the information about higher-level selection can be represented in the so-called selection coefficients of organisms or genes (Levins 1970, 1975; Wade 1979), but that simply does not imply that, in nature, it is individual or genic selection which is always occurring. Genes may be modelled as maximizing their fitnesses, but that leaves open the question of what causal processes propel changes in gene frequencies (Wilson 1980). 
Earlier, I commented on the fact that the interdefinability of fitness values and selection coefficients should not mislead us into thinking that fitness and selection are essentially equivalent concepts. The same point applies here: it is essential not to confuse facts about the mathematics of our models with facts about the causal structure of the processes modelled. It is desirable, of course, that our models be realistic. But it is a naive realism which thinks that every biologically interesting distinction will be forced on us by the exigencies of mathematical modelling.

One last confusion which I hope this discussion lays to rest is that between the issue of the unit of selection and the issue of the unit of replication (see Hull 1980 and 1981 for discussion). Group selectionists do not deny that the gene is the mechanism by which biological objects pass on their characteristics; the issue of cultural evolution is not an issue here. But this shared assumption about the unit of replication simply cuts no ice. That genes are passed along leaves open the question of what causes their differential transmission (pace Dawkins 1976). This is not to say that facts about heritability are irrelevant to the question of how selection at different levels may produce cumulative evolution (see Lewontin 1970 for this kind of argument). But any such argument must do more than merely point out that genes are the devices by which characteristics are inherited.

\section{Between Scylla and Charybdis}

The stock market crash, which was a social fact, was caused by market conditions, which constitute other social facts. These market conditions, in turn, were caused by individual interactions. By transitivity of causality, the individual interactions caused the stock market crash. If social facts cause something, so do individual facts. Once we decide to avoid atomism on the one hand and hypostatis on the other, the sensible middle course appears to provide no asymmetry between the social and the individual; both are causally efficacious.

Yet, it is emphatically not the case that if group selection causes something, so does individual selection. Group selection and individual selection are objectively distinct forces. Individual selection does not require an atomistic view of the organism; it does not require one to ignore the fact that organismic fitness is context sensitive. Individual selection is a process that a sensible individualist can embrace. Similarly, group selection does not require a reification of the group; it does not force one to suppose that groups are something above and beyond the interactions of their member individuals and the environment. Group selection is a process that a sensible holist can embrace. And, best of all, it is a substantive empirical question what the role and importance of these two forms of selection has been in the history of evolution.

Holism and individualism in the social sciences should have such luck. To move beyond truisms to nontrivial empirical issues, holists and individualists need to formulate their dispute with reference to 
specific social forces. Although there is no real question involved in asking whether a certain evolutionary outcome was caused by properties of groups or by properties of individuals, there is a substantive question involved in asking whether that outcome resulted from individual selection or from group selection. In just the same way, societies change because of the way their constituent groups interact, and these groups, ultimately, are caused to be the way they are by the individuals they contain. The debate between holism and individualism might become fruitful if specific mechanisms were considered and the question were then posed with respect to them: does their impact on individuals correspond to the causal structure we have identified in group selection processes, or does their activity represent a form of individual selection? Although it is probably a mistake to try to mimic the units of selection debate too closely, and there is no reason why the holism/individualism controversy must be recast in its terms, let's explore, in conclusion, what individual and group selection would look like in the case of social processes mediated by cultural, rather than genetic, evolution.

Social institutions can be viewed as selection mechanisms. They discriminate among individuals in virtue of their having certain properties and differentially distribute effects on that basis. This description encompasses a great many, diverse, social processes and appears to be nontendentious, in that it is consistent with the outlook of neoclassical and Marxist social thought alike.

As an example, consider a college admissions test. The test discriminates among individuals, and, on the basis of that discrimination, the individual is either admitted or not to a particular college. Is this a case of individual or of group selection? The test tests individuals, of course, but that doesn't show that it embodies a kind of individual selection. And individuals are influenced in their ability to do well on the test by the groups to which they belong. But that doesn't show that the test is a form of group selection. As we have seen earlier, neither the fact that individuals are the material basis of groups nor the fact that individual properties are context sensitive suffices to decide the issue between individual and group selection.

The overall ability to do well on the test can presumably be broken down into a number of component abilities. Are there component abilities which an individual has, simply in virtue of its belonging to a group of some particular kind? Are the properties of the group which have this effect on individual ability the result of interaction among individuals? Could two individuals who are otherwise similar differ in ability simply in virtue of their belonging to different groups? Could two individuals who are otherwise quite different possess the same component ability simply in virtue of their belonging to the same group? If the answers to these questions are yes, then the admissions test would appear to have the earmarks of a mechanism of group selection.

Although each of us probably thinks that he or she can readily answer the above questions, a note of caution is in order. The 
mechanisms of selection processes are often difficult to discern, and it is a mistake to think that one can conclusively identify the character of a mechanism from the kinds of results it produces. Perhaps the admissions test gives greater than proportional representation to some particular group; it doesn't follow that the test involves group selection for membership in that group. In this case, a serious assessment of what the test is doing must be based on a serious understanding of the various abilities that affect the ability to do well on the test. Characterizing these components is a highly nontrivial task, one which we have barely begun to discharge. Although it is transparent that the admissions test is a form of selection, the character of this selection process is in many ways extremely opaque.

Another application to social processes that can be made of our distinction between group and individual selection involves the idea of the selection of selection processes. Besides wanting to answer the question of how the admissions test works, one would also like to know where it came from -- how it came to be used as the admissions test. Even if it were true that the admissions test embodied a form of individual selection, the possibility remains open that it evolved by a process of group selection. Perhaps part of the cause of its being used is that it has certain group level results; this may be true even if the test does not make its discriminations on the basis of group membership.

Marxist critiques of "bourgeois" social science often have two components (Keat and Urry 1977). First, bourgeois social science is allegedly too individualistic in its orientation, seeing the individual rather than the group as the correct unit of analysis. Secondly, it is claimed to be superficial in the kinds of questions it asks about society, typically focusing on issues concerning the regularities that social institutions obey, rather than on more structural questions having to do with why those institutions are as they are. These two lines of criticism are not unrelated, of course, since, for Marxists, an explanation of why particular social institutions have the form they do must crucially involve considerations of class conflict. From this point of view, the results of bourgeois social theory need not be false, but they must be incomplete. This means that if they are, mistakenly, taken to be complete, they will offer a distorted view of social life.

One might interpret this point of view as holding that social institutions, at least in bourgeois society, embody a form of individual selection, but that they evolved by a process of group selection. One of the differences between bourgeois and feudal society may consist in which properties of individuals determine how social institutions treat them. Whereas membership in particular social groups was used to decide all manner of social sortings out, these criteria are much less often the ones which are directly invoked in bourgeois society. Rather, the mechanisms have shifted toward the structure of individual selection. But this by no means implies that those social institutions do not themselves constitute a form of class interest, since they may have evolved by a process of group selection. From this point of view, 
holism and individualism may each be correct in a limited domain, if each is understood as claiming that certain sorts of selection processes are at work in a given society.

Although this articulation of the holism versus individualism debate is not the only one possible, it does have one virtue. It yields a conception of individualism which is untainted by atomism and a conception of holism which is unspoiled by hypostatis. In so doing, it turns the social science dispute into what it ought to be -- a question about the character of social causation which is not decidable by a priori argument but can only be addressed by the assessment of evidence and the development of theories which are specifically sociological. This reformulation makes the dispute harder than it was before; the road away from truisms and toward contentful hypotheses about causal mechanisms is never an easy one. But this presumably is a price that an explanatory science willingly pays.

\section{Notes}

${ }^{1}$ I am very grateful to James Crow, David Hull, Richard Lewontin, and William Wimsatt. Discussions with them have been invaluable to me in developing my ideas on evolutionary theory in general and on group selection in particular. The research discussed here was supported by the John Simon Guggenheim Foundation and by the Graduate School of the University of Wisconsin, Madison. I also wish to thank the Museum of Comparative Zoology, Harvard University, for its hospitality during 1980-81.

2 The argument presented in Putnam (1975) and Fodor (1976) against identifying psychological and physical properties may be applicable to the relationship between social properties and the properties of individual psychology. Just as a given psychological property may be "multiply realizable" in indefinitely many physical forms, so a given social property may have indefinitely many realizations at the level of individual psychology. For an application of this line of thinking to the relationship of biological properties like fitness to physical properties, see Rosenberg (1978).

3 The present discussion of holism and individualism and of causality assumes the truth of determinism, but this assumption is not essential to the points at issue. If quantum mechanical states at one time do not uniquely determine such states at a later time, then, on the assumption that macro-states are token/token identical with quantum mechanical states, it follows that macro-states at one time do not determine macro-states at a later time. Thus, from the point of view of causal determination, not only will facts about individuals fail to causally determine social facts; it will also be true that earlier social facts fail to causally determine later social facts. So, if both holism and individualism are construed as making claims which imply that social facts are causally determined (but disagree about what does 
the determining), then both are mistaken. However, there still is scope for two other issues to be raised. First, if causality does not require causal determination (as in the theories of causality of Dretske and Snyder 1972 and of Mackie 1974), then it still is possible for social facts to be caused by individual facts, and for social facts to cause other social facts, as required above. Secondly, besides the question of the causal connections between and within levels, there is the possibility of identity relations obtaining between social and individual facts (and between macro- and micro-facts generally). This second possibility is enough to allow the holism and individualism issue to be addressed, even if the question of causality is set to one side.

4 It is sometimes remarked that for selection to act on a set of objects, the objects must share a "common environment." I take it that this concept of a common environment is not definable in terms of spatio-temporal proximity, but will involve the idea that some causal influence affects the objects involved. (This includes, of course, the idea that they affect each other.) Even so, the requirement of a common environment still appears to represent too stringent a demand on the concept of natural selection. For consider again two prey populations which are geographically isolated from each other; suppose that they are preyed upon by two different populations of predators. If the two prey populations are conspecific, and the two predator populations are too, it may be appropriate to view the individuals in both prey populations as participating in a single selection process. The objects in a single selection process must be acted on by agents which are qualitatively similar, not necessarily numerically identical.

5 In this paper, I will construe Williams' artefact argument as favoring individual selection hypotheses at the expense of group selection hypotheses. This does less than full justice to Williams' considered position, in which genic, rather than organismic, selection is the preferred level. However, a detailed discussion of genic selection must be postponed for another occasion.

6 In the light of this argument, consider the common definition of group selection reported in Wade's (1978) review article: "Group selection is defined as that process of genetic change which is caused by differential extinction or proliferation of groups of organisms." Note that, besides failing to distinguish group from individual selection, this definition, taken at its word, fails to distinguish group selection from drift.

${ }^{7}$ I take it that this point undermines the definition of group selection presented in Wimsatt (1980, p. 236): "A unit of selection is any entity for which there is heritable context-independent variance in fitness among entities at that level which does not appear as heritable contextindependent variance in fitness (and thus, for which the variance fitness is context-dependent) at any lower level of organization." As I understand it, this definition would imply that in our two pair-wise competitions between Arrowhead and Standard in one cage and between 
Chiricahua and standard in the other, we do not have a case of organismic, individual, selection. The reason is that the fitnesses of organisms in this situation are context-dependent.

8

For more refined definitions of altruism, see Wilson (1980).

9 Wade's fourth treatment he calls "random selection". Each canister is assigned a number and then a canister is chosen by picking a number at random. The chosen group is then used to found colonies of 16 until it is exhausted, at which point another canister is chosen at random. This is repeated until 48 next generation colonies are established. Although this process can be called "group selection", according to the definition of group selection used (see my footnote 6 above), it is not group selection, according to the definition to be presented in what follows. Moreover, if drift and selection are mutually distinct categories, it is hard to see how there could be such a thing as "random selection" at all.

${ }^{10}$ It is worth pointing out that if Wade's experiment provides genuine cases of group selection, then group selection need not involve groups with complex organizational properties or ones having especially intricate forms of sociality. Although interest in group selection sparked by the issue of altruism will naturally focus on such cases, this is not a consequence of the concept of group selection itself. Wade's group selection treatments selected for group properties which are absolutely universal when there are groups at all. of course, even though it is no problem finding groups which vary in size, it is not quite so inevitable that this variation is heritable. This further requirement, as noted in section 2, is needed if the selection process is to result in cumulative evolutionary change. 


\section{References}

Brodbeck, M. (ed.). (1968). Readings in Philosophy of the Social Sciences, New York: MacMillan.

Crow, J. (1979). "Genes that Violate Mendel's Rules." Scientific American 240(2): 134-146.

-..--- and Kimura, M. (1970). An Introduction to Population Genetics Theory. New York: Harper and Row.

Dawkins, R. (1976): The Selfish Gene, Oxford: Oxford University Press.

Dretske, F. and Snyder, A. (1972). "Causal Irregularity." Philosophy of Science 39: 69-71.

Fisher, R. (1930). The Genetical Theory of Natural Selection. New York: Dover.

Fodor, J. (1976). The Language of Thought. New York: Thomas Crowell.

Gould, S. (1980). "Is a New and General Theory of Evolution Emerging?" Paleobiology 6: 119-130.

Hull, D. (1980). "Individuality and Selection." Annual Review of Ecology and Systematics 11: 311-332.

-----. (1981). "The Herd as a Means." In PSA 1980, Volume 2. Edited by P.D. Asquith and R.N. Giere. East Lansing, Michigan: Philosophy of Science Associaton. Pages 73-92.

Keat, R. and Urry, J. (1977). Social Theory as Science, London: Routl edge and Kegan Paul.

Leven, H., Pavlovsky, O., and Dobzhansky, T. (1954). "Interaction of the Adaptive Values in Polymorphic Experimental Populations of Drosophila pseudoobscura." Evolution 8: 335-349.

Levins, R. (1970). "Extinction." In Some Mathematical Questions in Biology, Volume 2. Edited by M. Gerstenhaber. Providence, Rhode Isl and: American Mathematical Society. Pages 75-108.

--.--.. (1975). "Evolution in Communities Near Equilibrium." In Ecology and Evolution of Communities. Edited by M. Cody and J. Diamond. Cambridge, Massachusetts: Harvard University Press. Pages 16-50.

Lewontin, R. (1970). "The Units of Selection." Annual Review of Ecology and Systematics 1: 1-18.

169.

(1978). "Adaptation." Scientific American 229(3): 156- 
- (-2 and Dunn, R. (1960). "The Evolutionary Dynamics of a Polymorphism in the House Mouse." Genetics 45: 705-722.

Mackie, J. (1974). The Cement of the Universe, Oxford: Clarendon Press.

Mills, S. and Beatty, J. (1979). "The Propensity Interpretation of Fitness." Philosophy of Science 46: 263-286.

Putnam, H. (1967). "Psychological Predicates." In Art, Mind and Religion. Edited by W.H. Capitan and D.D. Merrill. Pittsburgh: University of Pittsburgh Press. Pages 37-48. (Reprinted as "The Nature of Mental States." In Mind, Language, and Reality. (Philosophical Papers, Volume 2.) Cambridge: Cambridge University Press, 1975. Pages 429-440.)

Rosenberg, A. (1978). "The Supervenience of Biological Concepts." Philosophy of Science 45: 368-386.

Sober, E. (1980). "Evolution, Population Thinking, and Essentialism." Philosophy of Science 47: 350-383.

-n-- (1981a). "Evolutionary Theory and the Ontological Status of Properties." Philosophical Studies 40: 147-176.

- (1981b). "The Principle of Parsinony." British Journal for the Philosophy of Science 32: 145-156.

Stanley, S. (1975). "A Theory of Evolution Above the Species Level." Proceedings of the National Academy of Science, USA 72: 646-650.

Wade, M. (1976). "Group Selection Among Laboratory Populations of Tribolium." Proceedings of the National Academy of Sciences, USA 73: $4604-4607$.

-.-. (1978). "A Critical Review of the Models of Group Selection." Quarterly Review of Biology 53: 101-114.

-1-- (1979). "The Evolution of Social Interactions by Family Selection." American Naturalist 113: 399-417.

Williams, G. (1966). Adaptation and Natural Selection. Princeton, New Jersey: Princeton University Press.

Wilson, D. (1980). The Natural Selection of Populations and Communities, Menlo Park, California: Benjamin/Cummings Publishing Co.

Wimsatt, W. (1980). "Reductionistic Research Strategies and Their Biases in the Units of Selection Controversy." In Scientific Discovery: Case Studies. Edited by T. Nickles. Dordrecht, North Holl and: D. Reidel Publishing Co. Pages 213-259.

Wright, S. (1931). "Evolution in Mendelian Populations." Genetics 16: $97-159$. 
------. (1978). Evolution and the Genetics of Populations. (Variability Within and Among Natural Populations, Vol ume IV.) Chicago: University of Chicago Press.

Wynne-Edwards, V. (1962). Animal Dispersion in Relation to Social Behavior. Edinburgh: Oliver and Boyd. 